\title{
ESTADO DE LA EMPRESA INDUSTRIAL: VISIÓN DE ESTUDIOS FORMALIZADOS
}

\section{STATE OF THE INDUSTRIAL ENTERPRISE: VISION OF FORMALIZED STUDIES}

Francisco Javier Cárcel Carrasco ${ }^{1}$

1. Doctor Ingeniero Industrial. Universitat Politècnica de València. Camino de Vera $S / N$, 4022, Valencia (España). E-mail: fracarc1@csa.upv.es

Citación sugerida:

Cárcel Carrasco, F.J. (2017). Estado de la empresa industrial: visión de estudios formalizados. $3 C$ Empresa: investigación y pensamiento crítico, 6(1), 22-36. DOI: $<$ http://dx.doi.org/10.17993/3cemp.2017.060129.22-36/>. 


\section{RESUMEN}

Mediante el análisis y la visión de estudios formalizados sobre las características fundamentales de la empresa industrial española, se pueden tomar conclusiones de cómo será su evolución y las tendencias que las forman. Para ello, se han resumido y analizado algunos de los estudios sectoriales que marcan la tendencia industrial, indicándonos sus carencias o principales características que las definen.

\section{ABSTRACT}

Through the analysis and the vision of studies made on the fundamental characteristics of the Spanish industrial company, conclusions can be taken of how their developments and trends that form them. For this purpose have been summarized and analyzed some of the sectoral studies that indicate the industry trend, indicating its shortcomings or main characteristics that define them.

\section{PALABRAS CLAVE}

Empresa industrial; estudios sectoriales; gestión de empresas.

\section{KEY WORDS}

Industrial enterprise; sectoral studies; management of companies. 


\section{INTRODUCCIÓN}

El sector empresarial español tiene unas características clásicas que es necesario analizar para una visión del conjunto. Tomando un análisis del funcionamiento de las empresas en el periodo anterior a la crisis española, el sector industrial español está constituido (Figura 1), según datos obtenidos del Directorio Central de Empresas (DIRCE) por más de 245.000 empresas (a 1 de enero de 2008), lo que representa un 7\% del total de empresas del directorio (la agricultura se queda fuera del ámbito poblacional del DIRCE). En la Tabla 1 se recoge el número de empresas del DIRCE distribuidas por ramas de actividad para los años 1999 y 2008. En ese decenio aumentó el número total de empresas así como la importancia relativa del sector de la construcción con respecto a los otros dos sectores: servicios e industria. En España, la mayoría de las empresas se dedican al sector servicios (78\%) mientras que a la industria se dedican, de media, sólo 7 de cada 100.

Tabla 1: Número de empresas por rama de actividad en España 2008.

\begin{tabular}{|c|c|c|c|c|c|}
\hline Actividad & 1999 & $\begin{array}{l}\% \text { sobre } \\
\text { total }\end{array}$ & 2008 & $\begin{array}{l}\% \text { sobre } \\
\text { total }\end{array}$ & $\begin{array}{l}\text { Variación } \\
\text { 2008/1999 (\%) }\end{array}$ \\
\hline TOTAL & 2.518 .801 & 100 & 3.422 .239 & 100 & 136 \\
\hline Industria & 237.782 & 9 & 245.588 & 7 & 103 \\
\hline Construcción & 271.616 & 11 & 501.056 & 15 & 184 \\
\hline Servicios & 2.009 .403 & 80 & 2.675 .595 & 78 & 133 \\
\hline
\end{tabular}

Fuente: Directorio Central de Empresas. INE

Fuente: (INE, 2008).

La Tabla 2 presenta un análisis más detallado del número de empresas de servicios, industria y construcción según el número de asalariados. Si bien las empresas industriales representan un $7 \%$ del total, en las que cuentan con 20 y más ocupados los porcentajes se mantienen superiores al $20 \%$ en todos los intervalos; es decir la importancia relativa de la industria aumenta con el tamaño de las empresas y alcanza un máximo en el intervalo de 20 a 100 ocupados, en el que una de cada cuatro empresas se dedica a la industria.

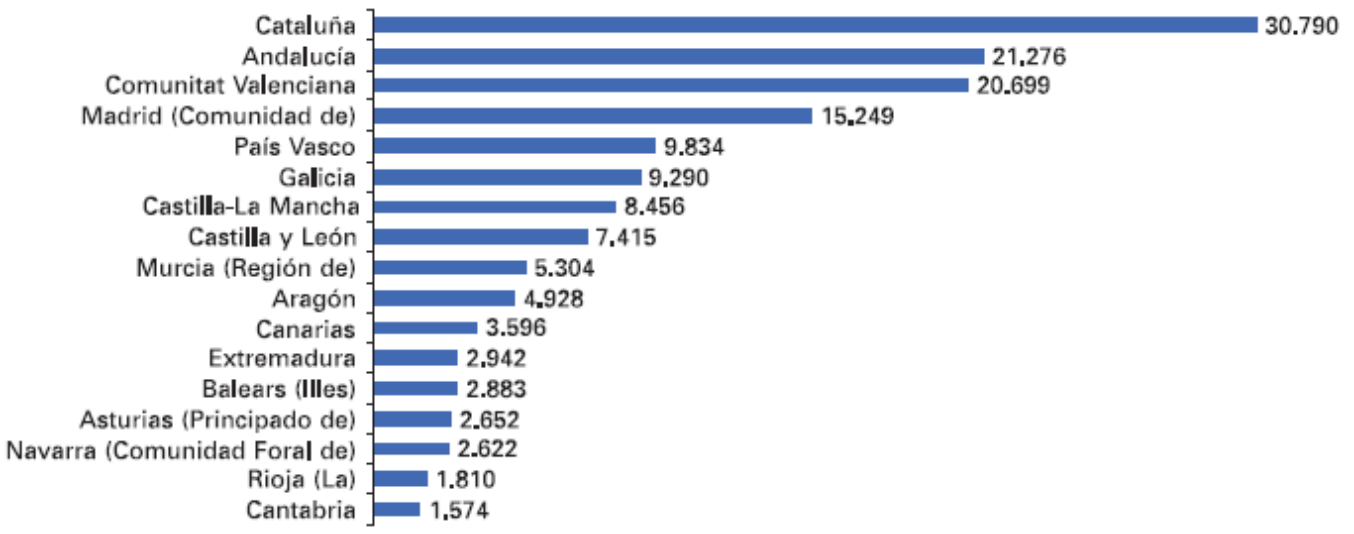

Figura 1: Número de empresas industriales por comunidad autónoma en España 2008.

Fuente: (INE, 2008). 
Tabla 2: Empresas por actividad y tamaño en España 2008.

\begin{tabular}{lcccc} 
(Porcentaje) & Industria & & \multicolumn{1}{c}{ Construcción } & \multicolumn{2}{c}{ Servicios } \\
\hline TOTAL & 7 & 15 & 78 \\
\hline Sin asalariados & 5 & 13 & 82 \\
De 1 a 9 & 8 & 15 & 77 \\
De 10 a 19 & 19 & 23 & 58 \\
De 20 a 49 & 25 & 23 & 51 \\
De 50 a 99 & 25 & 18 & 56 \\
\hline De 100 a 499 & 23 & 15 & 62 \\
500 y más & 20 & 7 & 72 \\
\hline
\end{tabular}

Fuente: Directorio Central de Empresas. INE

Fuente: (INE, 2008).

\section{ESTUDIOS FORMALIZADOS DEL ESTADO DE LA EMPRESA INDUSTRIAL}

Para marcar la importancia de la industria española en el conjunto de la Unión Europea, se pueden extraer los datos de la Tabla 3. Los datos europeos de Contabilidad Nacional referidos al periodo 1997-2007 muestran una evolución similar al caso español: aumento de los sectores de la construcción y de los servicios y una paulatina disminución de la importancia de la industria europea en el total de la economía. Si en 1997 esa participación era del $23,3 \%$, en 2007 ha pasado a ser del $20,2 \%$. En el mismo periodo el sector agrícola ha disminuido del $2,8 \%$ al $1,8 \%$.

La heterogeneidad en la composición sectorial de los veintisiete países que integran la UE se visualiza en la Tabla 3. Hay siete países en los que la industria aporta la cuarta parte del valor añadido de sus respectivas economías: Alemania, Eslovaquia, Eslovenia, Finlandia, Hungría, la República Checa y Rumanía. En el resto la participación de la industria está por debajo de esa cifra y llega a ser inferior al $10 \%$ en Chipre y Luxemburgo.

De estos datos se puede ver la evolución de la participación industrial en España y en el conjunto de la Unión Europea, así como la de los dos países en los que la industria tenía en 1997 la mayor y la menor importancia en su propia economía (Irlanda y Chipre respectivamente) y el país que más aporta al sector industrial en la UE (Alemania) y que además, junto con Eslovaquia, son los dos únicos países en los que la participación del sector industrial en el valor añadido total respectivo, sigue una tendencia alcista. 
Tabla 3: Participación de cada rama en el valor añadido de la UE-27. 2007.

\begin{tabular}{|c|c|c|c|c|}
\hline & $\begin{array}{l}\text { Indus- } \\
\text { tria }\end{array}$ & $\begin{array}{l}\text { Agricul- } \\
\text { tura }\end{array}$ & $\begin{array}{l}\text { Cons- } \\
\text { trucción }\end{array}$ & $\begin{array}{l}\text { Servi- } \\
\text { cios }\end{array}$ \\
\hline UE-27 & 20,2 & 1,8 & 6,4 & 71,6 \\
\hline República Checa & 32,6 & 2,4 & 6,3 & 58,7 \\
\hline Eslovaquia & 31,3 & 3,5 & 7,9 & 57,2 \\
\hline Rumanía & 27 & 6,4 & 10,1 & 56,6 \\
\hline Alemania & 26,4 & 0,9 & 4 & 68,7 \\
\hline Eslovenia & 26,4 & 2,4 & 8 & 63,3 \\
\hline Finlandia & 26,2 & 3,2 & 6,4 & 64,1 \\
\hline Hungría & 25,1 & 4 & 4,7 & 66,4 \\
\hline Polonia & 24,5 & 4,3 & 7,3 & 63,9 \\
\hline Bulgaría & 24,1 & 6,2 & 8,2 & 61,5 \\
\hline Irlanda & 23,7 & 1,7 & 9,9 & 64,8 \\
\hline Austria & 23,5 & 1,8 & 7,1 & 67,7 \\
\hline Suecia & 23,4 & 1,4 & 4,9 & 70,2 \\
\hline Lituania & 22,6 & 4,5 & 10,2 & 62,7 \\
\hline Italia & 21,4 & 2,1 & 6,1 & 70,4 \\
\hline Estonia & 21,3 & 2,8 & 9,1 & 66,8 \\
\hline Dinamarca & 20,3 & 1,2 & 6,1 & 72,4 \\
\hline Países Bajos & 18,8 & 2 & 5,6 & 73,6 \\
\hline Bélgica & 18,7 & 0,8 & 5,3 & 75,2 \\
\hline Malta & 18,3 & 2,6 & 3,6 & 75,5 \\
\hline Portugal & 18 & 2,5 & 6,5 & 73,1 \\
\hline España & 17,5 & 2,9 & 12,3 & 67,4 \\
\hline Reino Unido & 16,7 & 0,6 & 6,4 & 76,3 \\
\hline Letonia & 14,2 & 3,6 & 9 & 73,2 \\
\hline Francia & 14,1 & 2,2 & 6,3 & 77,4 \\
\hline Grecia & 13,3 & 3,8 & 7 & 75,9 \\
\hline Chipre & 9,8 & 2,2 & 9,1 & 78,9 \\
\hline Luxemburgo & 9,8 & 0,4 & 5,8 & 84 \\
\hline
\end{tabular}

Fuente: (Eurostar, INE, 2008).

Para ver la perspectiva de los sectores industriales a nivel mundial, se pueden observar los datos proporcionados por la OCDE que se presentan en la Tabla 4, confirman los cambios estructurales mencionados con anterioridad: la paulatina disminución de la participación de la industria manufacturera en la economía de la mayor parte de los países.

La tabla presenta la distribución porcentual por sectores del valor añadido de cada país, distinguiendo entre agricultura, industria y construcción y sector servicios y permite comparar datos relativos a 2007 y 1997. En el periodo 1997-2007 la participación de la agricultura ha disminuido en todos los países; en cuanto al sector servicios los porcentajes han aumentado en todos, salvo en Canadá, Noruega y Australia. En la tabla están agrupados los sectores de la industria y la construcción e incluye datos porcentuales tanto para ese total como para el subconjunto de la industria manufacturera, lo que permite confirmar que es en esta última en dónde la disminución porcentual es más acusada. Del total de países analizados, sólo en Suiza y Corea aumenta la aportación de las manufacturas, mientras que son cinco los países que experimentan incrementos en la participación del total de la industriaconstrucción (Australia, Canadá, España, México y Noruega). 
En el caso particular de España, si bien la participación total de la industria-construcción aumenta medio punto porcentual, el conjunto de la actividad manufacturera disminuye 3,8 puntos porcentuales descendiendo de un $19 \%$ a un $15,2 \%$.

Tabla 4: Participación de cada rama en el valor añadido de la UE-27. 2007.

\begin{tabular}{|c|c|c|c|c|c|c|c|c|c|}
\hline \multirow[t]{3}{*}{ País } & \multicolumn{3}{|c|}{$\begin{array}{l}\text { Agricultura } \\
\% \text { del valor añadido }\end{array}$} & \multicolumn{4}{|c|}{$\begin{array}{l}\text { Industria y construcción } \\
\% \text { del valor añadido }\end{array}$} & \multicolumn{2}{|c|}{$\begin{array}{l}\text { Servicios } \\
\% \text { del valor añadido }\end{array}$} \\
\hline & \multirow{2}{*}{\multicolumn{2}{|c|}{2007}} & \multirow[b]{2}{*}{1997} & \multicolumn{2}{|l|}{ Total } & \multicolumn{2}{|c|}{ Manufacturera } & \multirow[b]{2}{*}{2007} & \multirow[b]{2}{*}{1997} \\
\hline & & & & 2007 & 1997 & 2007 & 1997 & & \\
\hline Canadá & $(-3)$ & 2,2 & 2,5 & $(-3) 31,7$ & 30,9 & $(-3) 16,2$ & 18,0 & $(-3) 66,1$ & 66,6 \\
\hline Estados Unidos ${ }^{2}$ & & 1,3 & 1,7 & 21,8 & 25,5 & 13,3 & 1. 17,3 & 76,9 & 72,8 \\
\hline México & $(-1)$ & 3,3 & 5,5 & $(-1) 35,8$ & 35,2 & $(-1) 18,9$ & 21,4 & $(-1) 60,9$ & 59,2 \\
\hline Islandia ${ }^{2}$ & $(-2)$ & 5,8 & 9,8 & $(-2) 23,7$ & 28,9 & $(-2) 10,1$ & 16,4 & $(-2) 70,5$ & 61,3 \\
\hline Suiza & & 1,2 & 1,8 & 28,0 & 28,5 & 20,3 & 20,1 & 70,8 & 69,8 \\
\hline Noruega & & 1,4 & 2,4 & 42,7 & 37,1 & 10,4 & 12,3 & 55,9 & 60,4 \\
\hline España & & 2,9 & 5,0 & 29,8 & 29,3 & 15,2 & 19,0 & 67,4 & 65,7 \\
\hline Turquía & & 8,7 & 10,8 & 27,8 & 37,2 & 18,7 & 27,9 & 63,5 & 52,2 \\
\hline Corea & & 2,9 & 5,2 & 37,1 & 37,9 & 27,6 & 25,6 & 60,0 & 56,2 \\
\hline Japón ${ }^{3}$ & & 1,4 & 1,7 & 28,5 & 32,8 & 20,6 & 22,2 & 70,1 & 65,5 \\
\hline Australia & & 2,6 & 3,4 & 29,1 & 27,7 & 10,5 & 14,3 & 68,4 & 68,9 \\
\hline Nueva Zelanda ${ }^{4}$ & $(-3)$ & 6,2 & 6,8 & $(-3) 24,6$ & 25,5 & (-3) 15,3 & 16,9 & $(-3) 69,2$ & 67,6 \\
\hline
\end{tabular}

1. Según el Sistema Nacional de cuentas para el año 1993 y la Clasificación Industrial Estandar Internacional (ISIC), Revisión 3 (1990). Valor añadido estimado a precios básicos incluye el FISIM

2. Valor añadido estimado al coste de los factores

3. Valor añadido estimado aproximadamente a precios de mercado

4. Valor añadido estimado a precios de productor

- $n$. Las cifras en estas celdas son de años anteriores (posteriores, si $+n$ ) al año de referencia. Por ejemplo si la columna se refiere a 2007, una celda con ' -1 ' hace referencia a 2006

Fuente: La OCDE en cifras 2009. OCDE

Fuente: (Eurostar, INE, 2008).

\section{OTROS FACTORES DE INCIDENCIA}

El estudio de los gastos de explotación, una vez descritas y comentadas las distintas componentes de los ingresos, permite completar, en una primera aproximación, el análisis de la actividad productiva de las empresas.

La distribución de las partidas que constituyen los gastos de explotación aparece reflejados en la Tabla 5.

Se pone de relieve que los consumos son, sin duda, la componente más importante de la estructura de gastos, con un porcentaje de participación sobre el total superior al doble de la suma de los porcentajes del resto de componentes.

Si se analiza la estructura de los gastos a nivel de agrupaciones de actividad, se observa que los consumos se mantienen en todas ellas como la componente más importante. No obstante, los porcentajes son lógicamente variables de unas actividades a otras, por lo que aumenta, en determinadas agrupaciones, la importancia relativa de las otras componentes de gasto. En concreto destacar que, en cuatro agrupaciones de actividad, los gastos de personal llegan a superar el $20 \%$ de los gastos totales de explotación: en la industria textil, 
confección, cuero y calzado (21\%); papel, edición, artes gráficas y reproducción de soportes grabados (22\%); maquinaria y equipo mecánico (21\%); y en industrias manufactureras diversas (23\%). A parte de la variable de gastos de personal, las otras dos componentes más relevantes del gasto son: los consumos y los servicios exteriores.

El concepto global de consumos y trabajos realizados por otras empresas incluye tanto el consumo de materias primas (bienes adquiridos para su transformación en el proceso productivo), como el de otros aprovisionamientos (combustibles, carburantes, repuestos, embalajes, material de oficina, etc.) y el de mercaderías (bienes adquiridos para revenderlos sin someterlos a un proceso de transformación), así como el gasto correspondiente al trabajo que, formando parte del proceso de producción propia, se encarga y es realizado por otras empresas.

El análisis interno de la variable consumos, muestra que el consumo de materias primas representa, en media, el $66 \%$ de la cifra total, siendo el componente mayoritario en todas las agrupaciones de actividad.

Tabla 5: Gastos de explotación en las empresas. 2007.

\begin{tabular}{|c|c|c|}
\hline & $\begin{array}{l}\text { Millones } \\
\text { de euros }\end{array}$ & $\begin{array}{l}\% \text { sobre } \\
\text { el total }\end{array}$ \\
\hline Consumos y trabajos realizados por otras empresas & 400.621 & 67,7 \\
\hline Gastos de personal & 83.206 & 14,1 \\
\hline Servicios exteriores & 84.628 & 14,3 \\
\hline Dotaciones para amortización del inmovilizado & 22.999 & 3,9 \\
\hline
\end{tabular}

Fuente:(INE, 2008).

Dentro del total de gastos de explotación, los servicios exteriores representan, en media, un $14,3 \%$ del total, lo que supone una cifra muy semejante a la correspondiente a los gastos de personal (14,1\%). No obstante, su importancia sobre el total de gastos de explotación varía según la agrupación de actividad considerada, fluctuando desde el $8 \%$ de las industrias extractivas y del petróleo, hasta el $23 \%$ de la industria del papel, edición, artes gráficas y reproducción de soportes grabados.

La distribución porcentual de los servicios exteriores entre sus distintas componentes de gasto se presenta en la Tabla 6 . Los datos de la tabla van referidos a empresas con 20 y más trabajadores, y ponen de manifiesto la importancia en el desarrollo de la actividad de las empresas de determinados servicios tales como el transporte, los suministros, las reparaciones y los servicios de profesionales independientes. 
Tabla 6: Servicios exteriores en las empresas, 2007.

\begin{tabular}{lrrr}
\hline & $\begin{array}{c}\text { Millones } \\
\text { de euros }\end{array}$ & \multicolumn{2}{c}{$\begin{array}{l}\text { \% sobre } \\
\text { el total }\end{array}$} \\
\cline { 2 - 4 } Transportes & 12.645 & 16,8 \\
Suministros & 11.234 & & 14,9 \\
Reparaciones y conservación & 8.928 & & 11,9 \\
Servicios de profesionales independientes & 8.410 & & 11,2 \\
Publicidad, propaganda y relaciones públicas & 7.915 & 10,5 \\
\hline Arrendamientos y cánones & 7.346 & 9,8 \\
Primas de seguros & 1.558 & 2,1 \\
Gastos en I+D del ejercicio & 1.071 & 1,4 \\
Servicios bancarios y similares & 699 & 0,9 \\
Otros servicios & 15.456 & 20,5 \\
\hline
\end{tabular}

Nota: datos referidos a empresas con 20 y más personas ocupadas

Fuente: (INE, 2008).

El empleo, los costes de personal y el nivel de salarios constituyen, en conjunto, un atractivo campo de estudio dentro del análisis general de la estructura industrial. No sólo por sus implicaciones económicas y sus repercusiones sobre el volumen de gastos, sino también por la componente social que a ellos va asociada.

Los costes de personal han experimentado durante ese mismo periodo una progresiva pérdida de importancia en relación con el total de gastos de explotación de las empresas. En 1993 los gastos de personal suponían el 22\% del total de gastos, porcentaje que se ha ido reduciendo paulatinamente hasta alcanzar el $14 \%$ en 2007. En la figura 2 presenta tanto la evolución de los gastos de personal por ocupado (creciente al incorporar el efecto de la inflación) como la del ratio gastos de personal sobre gastos de explotación, poniéndose de relieve el descenso en 8 puntos porcentuales del mismo.

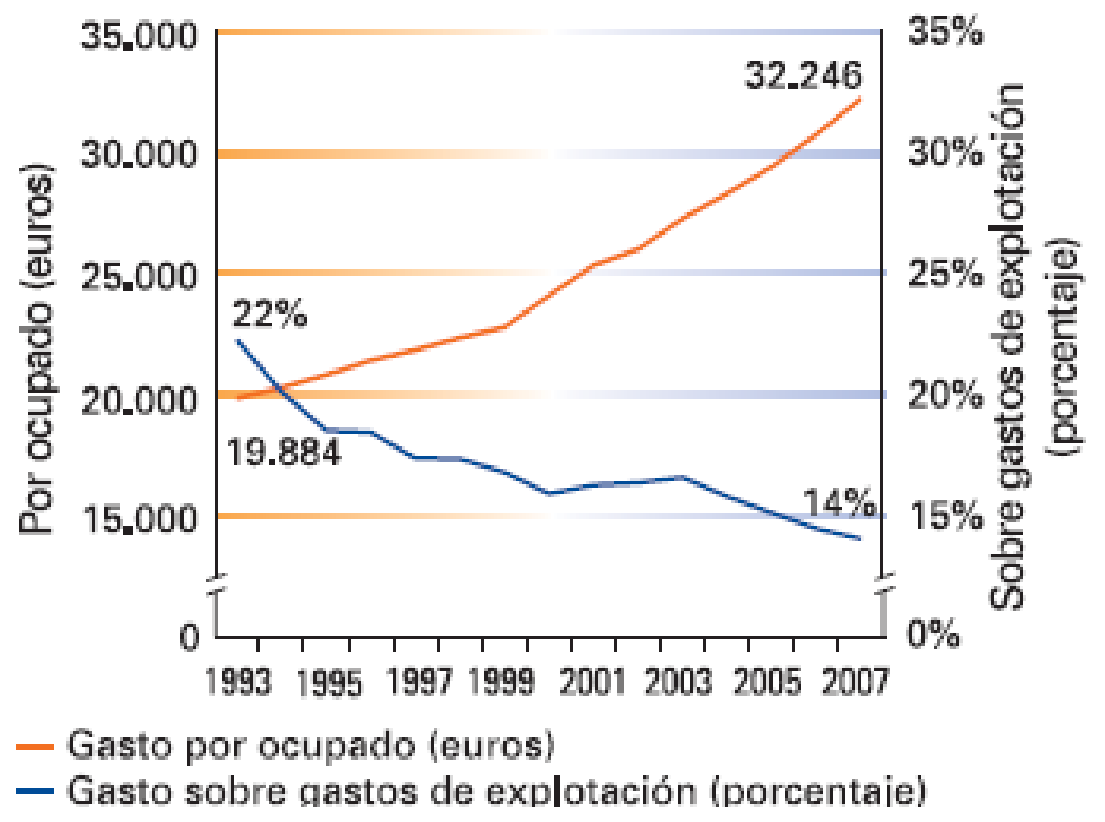

Figura 2: Gastos de personal en la industria en España 2008.

Fuente: (INE,2008). 
El sueldo por persona asalariada en el sector industrial español fue en 2007 de 25.127 euros. Dentro de los sectores, la agrupación de mayor salario, energía y agua, supera en más del doble a la agrupación de menor salario medio, la industria textil, confección, cuero y calzado.

Desde otro punto de vista, en el ámbito europeo efectuamos una comparación de la importancia relativa de los costes de personal a través de dos ratios. El primero de ellos aparece en la figura 3 donde se representa la relación entre los costes de personal y los respectivos valores de producción. En 2007 y para la media europea los costes de personal supusieron un $15 \%$ del valor de la producción. Las cifras por países oscilan desde el $9 \%$ de Irlanda al 19\% de Chipre. España ocupa en esa escala el décimo-cuarto lugar con un $14 \%$.

La dimensión de la empresa y la rama de actividad son dos de los factores que influyen en la productividad industrial. La relación existente entre productividad y dimensión de la empresa (expresada en términos de número de personas ocupadas) se observa en la figura 4 , en el que se visualiza la productividad media por ocupado para cada uno de los distintos intervalos de tamaño en los que se clasifica a las empresas industriales.

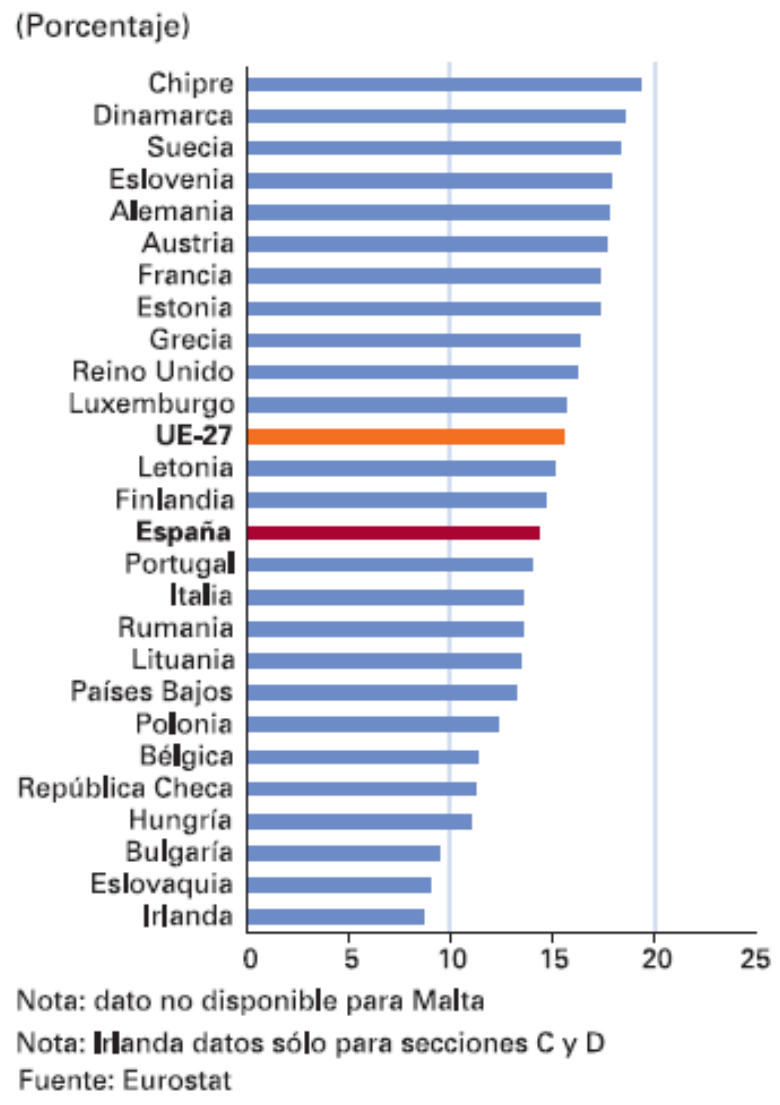

Figura 3: Costes de personal sobre valor de producción.

Fuente: (INE, 2008).

En la figura 4 se resalta el aumento progresivo de la productividad a medida que aumenta el tamaño de la empresa, así como las variaciones existentes en los incrementos de productividad de unos intervalos a otros. Por ejemplo, el porcentaje de variación entre los dos primeros intervalos correspondientes a las empresas de menor tamaño (6\%) se transforma en un $32 \%$ al calcular el incremento entre los dos de mayor tamaño. 
(Miles de euros)

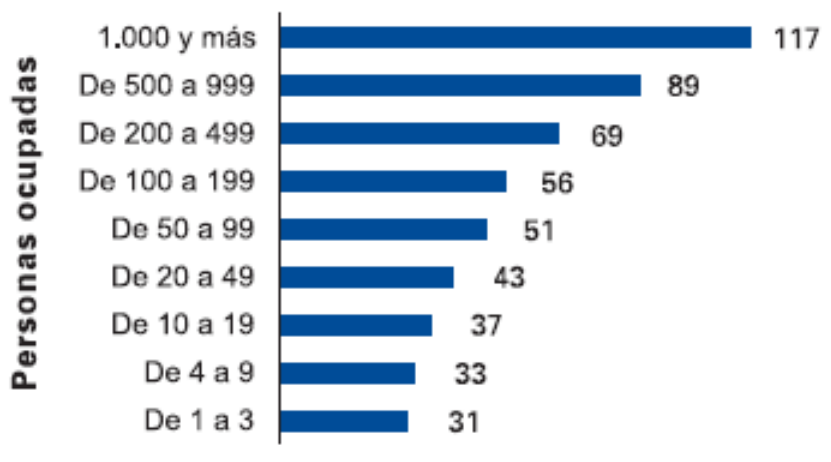

Figura 4: Productividad por ocupado y tamaño de la empresa en España.

Fuente: (INE, 2008).

Otro factor a tener en cuenta en el estudio de la productividad es la rama de actividad. Las diferencias de productividad, si se consideran los 3 grandes sectores industriales, son ya significativas (ver figura 5). A un mayor nivel de detalle en la desagregación de actividad, si se tienen en cuenta las catorce agrupaciones en las que se ha dividido la industria, se aprecian también notables diferencias de productividad entre las mismas (ver Tabla 7).

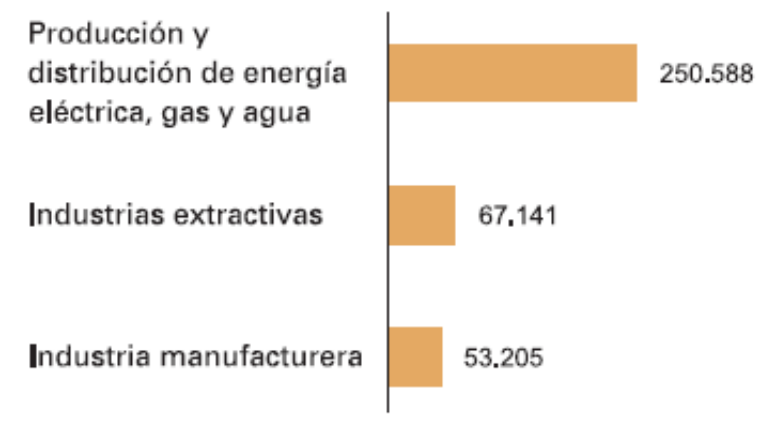

Figura 5: Productividad por ocupado y actividad de la empresa en España.

Fuente: (INE, 2008).

Tabla 7: Productividad por ocupado y agrupación actividad de la empresa en España.

\begin{tabular}{lr}
\hline Agrupación & \multicolumn{2}{l}{ Euros } \\
\cline { 2 - 2 } Energía y agua & 250.588 \\
Industrias extractivas y del petróleo & 159.927 \\
Industria química & 81.228 \\
Productos minerales no metálicos & 60.232 \\
Material de transporte & 59.625 \\
\hline Material y equipo eléctrico, electrónico y óptico & 57.413 \\
Papel, edición, artes gráficas y reproducción de soportes grabados & 54.635 \\
Alimentación, bebidas y tabaco & 52.173 \\
Metalurgia y fabricación de productos metálicos & 51.736 \\
Maquinaria y equipo mecánico & 50.930 \\
\hline Caucho y materias plásticas & 47.869 \\
Madera y corcho & 34.090 \\
Industrias manufactureras diversas & 32.388 \\
Industria textil, confección, cuero y calzado & 28.186 \\
\hline
\end{tabular}

Fuente: (INE, 2008).

La productividad por ocupado se incrementa a medida que aumenta el tamaño de las empresas. 
La productividad de las grandes empresas (con más de 1.000 ocupados) es más del triple de la correspondiente a las de menos de 20 ocupados.

La productividad por hora trabajada en el sector de energía y agua es superior a la de la industria extractiva y del petróleo y a la de la industria manufacturera.

Dentro de la industria manufacturera, la mayor productividad por ocupado se da en la industria química (81.228 euros), y la menor en la industria textil, confección, cuero y calzado.

\section{OTRAS CONSIDERACIONES EN LAS EMPRESAS}

\section{Características de la inversión en las empresas:}

En cuanto la inversión en activos materiales del sector industrial español fue en 2007 de 28.121 millones de euros, lo que representa un 4,5\% del total de la cifra de negocios de la industria. Según datos europeos del año 2007, España ocupa la posición undécima en cuanto a inversión bruta por ocupado con un dato de 11.477 euros.

A partir de los datos ofrecidos por la Encuesta Industrial de Empresas se puede analizar la estructura de la inversión y conocer asimismo la importancia que tiene cada uno de sus componentes determinando su participación en la cuantía total.

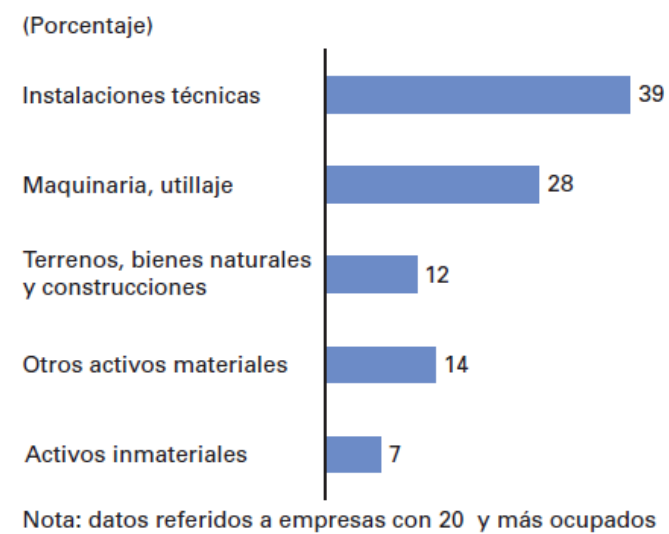

Figura 6: Componentes de la inversión en las empresas.

Fuente: (INE, 2008).

Se observa (figura 6) que la inversión conjunta en instalaciones y maquinaria es de una gran relevancia en la industria, constituyendo en 2007 el 67\% de la inversión total. Dentro del conjunto de otros activos materiales, (14\%) figuran incluidos los elementos de transporte (2\%) y los equipos informáticos (1\%). En cuanto a la rúbrica de terrenos, bienes naturales y construcciones, éstas últimas contribuyen con un $9 \%$ mientras que los terrenos y bienes naturales suponen el $3 \%$ restante.

Algunas de las conclusiones que se pueden extraer en cuanto la inversión en las empresas, es que el ratio de la inversión material por persona ocupada se incrementa a medida que aumenta el tamaño de la empresa. Las medianas y pequeñas empresas acumulan el $49 \%$ de la inversión en activos materiales. 


\section{Consumos energéticos en las empresas:}

Los datos de la Encuesta Industrial de Empresas permiten analizar la participación del gasto en consumos energéticos en el total de gastos de explotación, y en 2007 dicha participación es del 2,3\% para el conjunto de empresas con 20 y más personas ocupadas. Este porcentaje ha ido aumentando, en líneas generales, de una forma progresiva desde principios de la década, estimándose para el conjunto del periodo un incremento global en términos porcentuales del $20 \%$.

Una de las características relevantes en relación a esta variable es su fuerte dependencia de la rama de actividad considerada. Esto puede observarse a nivel de agrupación de actividad, y se manifiesta, en mayor medida, al nivel más detallado de sector de actividad. La Figura 7 presenta una distribución por agrupaciones de la importancia porcentual del consumo energético en relación al total de gastos de explotación y muestra la importancia de los consumos energéticos en algunas agrupaciones, como es la de productos minerales no metálicos, en la que llega a suponer un 7,5\% del total de gastos de explotación.

Ya se ha señalado que la importancia relativa de los consumos energéticos en las empresas depende, en gran medida, del sector de actividad al que pertenezcan. Los sectores en los que los consumos energéticos tienen una mayor repercusión sobre el gasto son los de fabricación de azulejos, baldosas, ladrillos, tejas y productos de tierras cocidas para la construcción (15\%); cemento, cal y yeso (14\%); pasta papelera, papel y cartón (12\%); fibras artificiales y sintéticas (10\%); acabado de textiles (10\%); y extracción de minerales no energéticos (10\%). En el extremo opuesto están sectores tales como los de edición; máquinas de oficina y equipos informáticos; motores eléctricos, trasformadores y generadores; aparatos de recepción, grabación y reproducción de sonido e imagen; vehículos de motor; y motocicletas, bicicletas y otro material de transporte en los que la participación de los consumos energéticos es inferior al $0,5 \%$ del total de gastos de explotación.

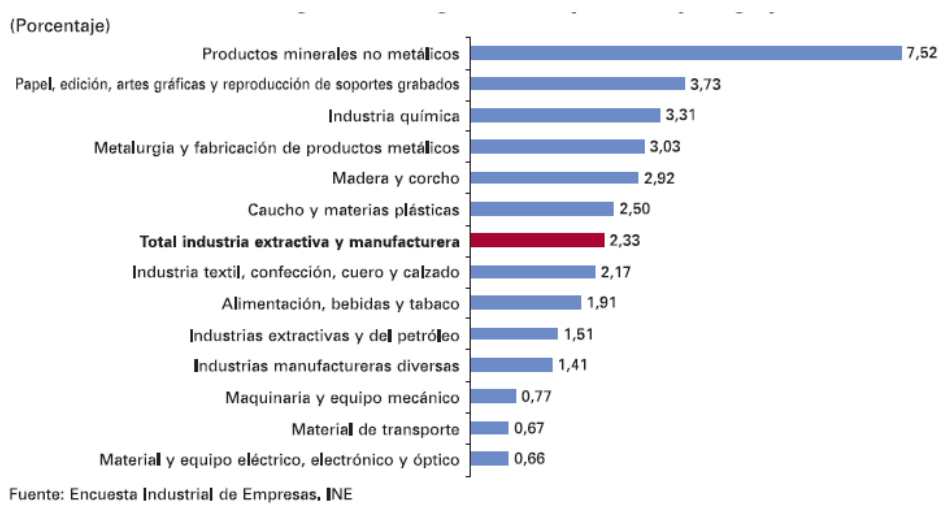

Figura 7: Consumos energéticos sobre gastos de explotación. Fuente: (INE, 2008). 
La Tabla 8 proporciona información sobre la importancia porcentual del consumo de cada tipo de combustible en el conjunto de la industria extractiva y manufacturera. La electricidad supone casi la mitad del consumo energético de las empresas industriales (48\%). El gas (28\%) y los productos petrolíferos (18\%) son los otros dos componentes, cuyo consumo es más relevante en el conjunto de la industria.

Tabla 8: Distribución de los consumos energéticos.

\begin{tabular}{|c|c|c|}
\hline Producto energético & $\begin{array}{l}\text { Valor } \\
\text { (miles } \\
\text { de euros) }\end{array}$ & $\begin{array}{l}\text { Porcentaje } \\
\text { sobre } \\
\text { el total }\end{array}$ \\
\hline Total & 10.980 .004 & 100 \\
\hline Electricidad & 5.302 .029 & 48 \\
\hline Gas & 3.047 .604 & 28 \\
\hline Productos petroliferos & 1.925 .778 & 18 \\
\hline Carbón y derivados & 295.188 & 3 \\
\hline Otros productos energéticos & 409.404 & 4 \\
\hline
\end{tabular}

Fuente: Encuesta de Consumos Energéticos. INE

Fuente: (INE, 2008).

En la Tabla 9 se presenta la distribución porcentual de los distintos consumos energéticos por agrupaciones de actividad.

Destaca la elevada participación porcentual del consumo de electricidad tanto en la industria del caucho y materias plásticas (77\%) como en la de material y equipo eléctrico (72\%) y la del consumo de gas en la industria química (43\%). Por otra parte, es también significativo el consumo de carbón y derivados en la industria de productos minerales no metálicos (12\%) en comparación con el resto de agrupaciones.

\begin{tabular}{|c|c|c|c|c|c|}
\hline Agrupación & Electricidad & Gas & & $\begin{array}{l}\text { Productos } \\
\text { petroliferos }\end{array}$ & $\begin{array}{l}\text { Carbón, } \\
\text { derivados y } \\
\text { otros productos } \\
\text { energéticos }\end{array}$ \\
\hline Industrias extractivas y del petróleo & 47 & 17 & 12 & 38 & 3 \\
\hline Alimentación, bebidas y tabaco & 49 & 19 & 24 & 24 & 3 \\
\hline Industria textil, confección, cuero y calzado & 55 & 5 & 28 & 14 & 3 \\
\hline Madera y corcho & 58 & 8 & 11 & 27 & 4 \\
\hline $\begin{array}{l}\text { Papel, edición, artes gráficas y reproducción de } \\
\text { soportes grabados }\end{array}$ & 46 & 6 & 38 & 8 & 7 \\
\hline Industria quimica & 37 & 7 & $\overline{43}$ & $\overline{11}$ & 8 \\
\hline Caucho y materias plásticas & 77 & 77 & 12 & 7 & 4 \\
\hline Productos minerales no metálicos & 32 & 2 & 36 & 20 & 12 \\
\hline Metalurgia y fabricación de productos metálicos & 61 & 1 & 22 & 13 & 5 \\
\hline Maquinaria y equipo mecánico & 57 & 7 & 15 & 26 & 2 \\
\hline Material y equipo eléctrico, electrónico y óptico & 72 & 2 & 9 & 17 & 1 \\
\hline Material de transporte & 62 & 2 & 20 & 15 & 4 \\
\hline Industrias manufactureras diversas & 58 & 8 & 6 & 31 & 5 \\
\hline
\end{tabular}

Tabla 9: Consumos energéticos por agrupación de actividad.

Fuente: (INE, 2008).

En cuanto a la energía, como conclusiones, se puede extraer que la electricidad supone casi

la mitad del consumo energético de las empresas industriales (48\%). El gas (28\%) y los 
productos petrolíferos (18\%) son los otros dos componentes, cuyo consumo es más relevante en el conjunto de la industria.

La electricidad es mayoritaria en todas las actividades salvo en la industria química y en la de productos minerales no metálicos, en las que el mayor consumo corresponde al gas. Los productos petrolíferos destacan por su importancia en extractivas y del petróleo; madera y corcho; maquinaria y equipo mecánico; e industrias manufactureras diversas. Por su parte, la industria de productos minerales no metálicos tiene un consumo significativo del carbón y sus derivados.

En los estudios presentados por la fundación Sepi (Sepi, 2009), sobre encuesta de estrategias empresariales sobre un estudio de una población de 1.798 empresas ( 1.336 de 200 y menos trabajadores y 462 de más de 200) se muestra como consecuencia de la crisis económica, su implicación en las empresas y en concreto en el sector industrial refleja el brusco ajuste de actividad. Si inicialmente su evolución estuvo marcada por el estallido de la burbuja inmobiliaria y el desplome de la actividad de la construcción, la rápida caída del consumo privado, de la inversión productiva y de la demanda externa terminó afectando profundamente a todas las ramas de actividad. Entre ellas, la fabricación de vehículos de motor, por su importancia en el del sector industrial español y sus efectos de arrastre, así como por su enorme relevancia en el conjunto de los intercambios comerciales con el exterior, constituye uno de los máximos exponentes del deterioro de la actividad productiva. Otras ramas, como las de alimentación, han podido aguantar mejor la recesión, aunque la generalidad de empresas se vio afectada por los problemas derivados de las restricciones crediticias, que fueron especialmente intensos en la segunda mitad del año 2008. La información de Contabilidad Nacional indica que la ralentización de la actividad industrial en 2007, con un crecimiento del valor añadido del 0,9\% (frente al 1,9\% del año anterior), se acentúa muy notablemente en 2008. Los datos señalan una reducción del $2,1 \%$ para el conjunto del año, contrastando el crecimiento interanual del 2,1\% del primer trimestre con la reducción del $-6,9 \%$ del cuarto trimestre. Los datos hasta ahora conocidos muestran que durante 2009 ha seguido registrándose un gran deterioro de la actividad industrial. Los datos acumulados de los tres primeros trimestres de 2009 reflejan una caída del VAB del sector industrial del $-16,1 \%$.

\section{CONCLUCIONES}

Analizando las encuestas sobre el estado de las empresas en España, con datos del INE y del SEPI, en España, la mayoría de las empresas se dedican al sector servicios (78\%) mientras que a la industria se dedican, de media, sólo 7 de cada 100. Se pone de relieve que los consumos son, sin duda, la componente más importante de la estructura de gastos, con un porcentaje de participación sobre el total superior al doble de la suma de los porcentajes del resto de componentes.

Si se analiza la estructura de los gastos a nivel de agrupaciones de actividad, se observa que los consumos se mantienen en todas ellas como la componente más importante. No 
obstante, los porcentajes son lógicamente variables de unas actividades a otras, por lo que aumenta, en determinadas agrupaciones, la importancia relativa de las otras componentes de gasto, como pueden ser los gastos de personal. La dimensión de la empresa y la rama de actividad son dos de los factores que influyen en la productividad industrial. La electricidad es mayoritaria en todas las actividades salvo en la industria química y en la de productos minerales no metálicos, en las que el mayor consumo corresponde al gas. Los productos petrolíferos destacan por su importancia en extractivas y del petróleo; madera y corcho; maquinaria y equipo mecánico; e industrias manufactureras diversas. Por su parte, la industria de productos minerales no metálicos tiene un consumo significativo del carbón y sus derivados.

En los estudios presentados por la fundación Sepi (Sepi, 2009), sobre encuesta de estrategias empresariales sobre un estudio de una población de 1.798 empresas (1.336 de 200 y menos trabajadores y 462 de más de 200) se muestra como consecuencia de la crisis económica, su implicación en las empresas y en concreto en el sector industrial refleja el brusco ajuste de actividad.

\section{REFERENCIAS BIBLIOGRÁFICAS}

INE. (2008). Instituto nacional de estadística. Panorámica de la industria. INE, Madrid.

Sepi. (2009). Fundación Sepi, Ministerio industria, turismo y comercio. Encuesta sobre estrategias empresariales. SEPI, Madrid. 